\title{
The impact of Replacement Conventional Dentures on Eating Experience
}

H.F. Al-Sultani ${ }^{1,2,3,4}$, J.C. Field ${ }^{5}$, J.M. Thomason ${ }^{1,2}$, P.J. Moynihan ${ }^{1,2,4}$

${ }^{1}$ Centre for Oral Health Research, Newcastle University, Newcastle upon Tyne, NE2 4BW, England, UK.

${ }^{2}$ School of Dental Science, Newcastle University, Newcastle Upon Tyne NE2 4BW, United Kingdom.

${ }^{3}$ Department of Paediatric, Orthodontics and Preventive Dentistry, Faculty of Dentistry/ University of Babylon, Hillah, Babel, IRAQ.

${ }^{4}$ Institute of Health and Society, Newcastle University, Newcastle upon Tyne, NE2 4BW, England, UK.

${ }^{5}$ School of Clinical Dentistry, University of Sheffield, Sheffield, S10 2TA, England, UK.

\section{Corresponding author}

\section{Professor Paula Moynihan}

School of Dental Sciences, Newcastle University, Framlington Place, Newcastle upon Tyne, NE2 4BW

Email: paula.moynihan@ncl.ac.uk

Telephone: +44 (0) 1912088241

Fax: 01912225928

Abstract word count: 300

Total word count (abstract to acknowledgments): 6780

Total number of tables/figures: 05

Number of references: 40

Keywords: edentulism, prosthesis, quality of life, eating behaviour, patient outcomes, cohort study. 


\section{Knowledge Transfer Statement:}

The results of this study improve the understanding of the impact of denture replacement on Eating Related Quality of Life (ERQoL). Clinicians are encouraged to pay more attention to the impact of wearing conventional complete dentures on social and emotional issues around eating. The findings should motivate clinicians and inspire specialists in Prosthodontics and oral rehabilitation to continue providing conventional complete dentures as a suitable treatment option for edentulous patients. 


\begin{abstract}
INTRODUCTION: Despite much research on the impact of edentulism and prosthetic rehabilitation on food and nutrient intake, there is little information on how replacing complete dentures impacts on social and emotional issues around eating.

OBJECTIVES: to investigate, in a cohort study, how replacing conventional complete dentures impacts on eating related quality of life (ERQoL). A secondary aim was to test the responsiveness of an Emotional and Social Issues Related to Eating (ESIRE) questionnaire to change in ERQoL.

METHODS: Participants, recruited from the Dental Hospital, Newcastle-upon-Tyne, UK, completed the self-administrated ESIRE questionnaire, before and after provision of new conventional complete dentures. Paired t-test was used to determine any change between preand post-treatment ESIRE scores, which can range from 0 (poor) to 100 (excellent). Cohen's $d$ effect size was used to measure the magnitude of change in ERQoL. Standardised Response Mean (SRM) was used to measure the responsiveness of the ESIRE questionnaire to changes in ERQoL.
\end{abstract}

RESULTS: 55 participants aged 52-85 years (mean 72 years), 21 males (42\%), and 29 females $(58 \%)$ completed the study. A statistically significant improvement in the total ESIRE scores was found, mean (SE) +20.3 (3.30), $p<0.001$. Equally, all domains of the ESIRE questionnaire showed significant improvements; enjoyment of food/ eating; +27.3 (3.63), $p<0.001$, self-consciousness/ embarrassment; +18.1 (3.88), $p<0.001$, interruption to meals; +13.3 (5.27), $p<0.05$, confidence when eating; +18.7 (4.84), $p<0.001$, time for eating/ preparation of meals; +18.5 (4.85), $p<0.001)$, and functional ability to eat; +18.2 (3.67), $p<0.001)$. Cohen's d was large (0.95) for the total score, and ranged from medium (0.37) to large (1.30) for all domains. Value of SRM was large (0.87) for the total score, and ranged from medium (0.36) to large (1.1) for all domains.

CONCLUSION: Denture replacement can directly improve ERQoL. The ESIRE questionnaire was responsive to clinically important changes in ERQoL. 


\section{Introduction}

It is acknowledged that the transition to edentulousness, and subsequent prosthetic rehabilitation, have various influences on overall Quality of Life (QoL), including oral and general health (Davis et al. 2000; Emami et al. 2013; Scott et al. 2006). Similarly, this transition has been shown to have a negative impact on the functional ability to consume different foods and nutrients (Moynihan et al. 2009). For the majority of edentulous patients, wearing conventional complete dentures is often the only available treatment option, predominantly due to their relatively low cost (Carlsson and Omar 2010). However, such a form of prosthetic rehabilitation could have several effects on patients' lives such as functional, structural and psycho-social influences and effects on Oral Health Related Quality of Life (OHRQoL), including eating (Basker et al. 2011; Davis et al. 2000; Forgie et al. 2005; Hyland et al. 2009; Müller 2014). Therefore, exploring patient perceptions regarding the effects of treatment options (e.g., conventional complete dentures) on health outcomes using specific patient-based tools or instruments is important.

OHRQoL measurements have been widely used to assess the impact of edentulousness and prosthetic rehabilitation on the life of edentulous individuals (AlBaker 2013; Ellis et al. 2010). Some of these instruments were used to measure the changes in OHRQoL, and patient satisfaction before and after denture relining (Krunić et al. 2015) or denture replacement (Kuo et al. 2013; Viola et al. 2013), and there were differences in the findings of these studies regarding the influence of denture replacement on the OHRQoL and patient's satisfaction. For example, several studies reported that provision of new complete dentures had a positive influence on patient satisfaction; however, it did not necessarily result in a significant social impact on OHRQoL (Forgie et al. 2005; Scott et al. 2006). Other studies, however, have shown an overall improvement (Ellis et al. 2007) or a significant improvement (Viola et al. 2013 ) in patient's satisfaction and OHRQoL among edentulous patients after provision of new conventional complete dentures. Whilst there is some understanding on the effect of denture replacement on patient satisfaction, and OHRQoL, little is known about the influence of denture replacement, particularly with conventional complete dentures, on social and emotional issues related to eating with dentures. For example, feelings and experiences of the patients during eating with their dentures, enjoyment of certain types of foods, especially in public arena with families or friends or how their dentures affect their social interaction with others. These factors are important in terms of clinical success; however, there is little in the literature regarding the effect of denture replacement on ERQoL. Therefore, exploring issues 
around eating with complete dentures is useful for two reasons; first, to know how to increase enjoyment of food/ eating among denture wearers, and second, to determine what needs to be done to change dietary behaviour to inform patient-centred care. In other words, there is a need to know about eating issues in order to provide patient-centred advice around eating that helps patients enjoy eating with dentures and help promote healthier eating (e.g., promoting eating vegetables and fruits in suitable ways for denture wearers). In order to fully understand the actual impact of wearing conventional complete dentures on ERQoL, researchers at Newcastle University, UK, have designed and validated a 24-item instrument to collect data on Social and Emotional Issues Related to the Eating (ESIRE questionnaire) (Kelly et al. 2012). The ESIRE questionnaire is a patient-based instrument designed with questions (quantitative part) to be answered using a Visual Analogue Scale (VAS) ranging from zero (anchored to a negative eating outcome) to 100 (anchored to a positive eating outcome). In addition, open questions (qualitative part) are included to be answered using free text. The qualitative data give detailed information or explanation to the breadth of data collected by VAS scale to further understand the effect of wearing conventional complete dentures on eating and enjoyment of food, and help explain any differences in responses to the VAS scale. The authors tested face validity, content validity and reliability tests. The responsiveness of an instrument developed primarily for measuring changes over time is important (Guyatt et al. 1987; Locker et al. 2004). Responsiveness of any instrument or questionnaire refers to the ability of a measure to determine or reflect change (e.g., improvements or deteriorations), which could happen within the selected sample (Guyatt et al. 1987). Therefore, the objective of this study was to apply the ESIRE questionnaire to a cohort study on patients requiring replacement dentures to determine any change in ERQoL, ERQoL through quantitative and qualitative investigation, before and after complete denture replacement. The primary aim was to determine if replacing complete dentures impacted on ERQoL. The null hypothesis $\left(\mathrm{H}_{0}\right)$ is there was no change in the average response of the ESIRE scores over the two time points. In other words, there would be no difference in the ERQoL, before and after treatment with new conventional complete dentures. The alternative hypothesis $\left(\mathrm{H}_{1}\right)$ was there is a change in the average response of the ESIRE scores over the two time points, in that, there would be a difference in the ERQoL, before and after treatment with new conventional complete dentures. The secondary aim was to measure responsiveness of the ESIRE questionnaire to measuring changes in ERQoL over time among this sample of complete denture wearers. 


\section{Methods}

\section{Study Design}

A prospective cohort study was conducted on patients (complete denture wearers) attending Newcastle Dental Hospital, Newcastle upon Tyne, UK from September 2015 to June 2016. All participants were assigned to undergraduate dental students under supervision for denture replacement, and according to the standard Newcastle Dental Hospital protocol. Inclusion criteria were edentulous patients with existing dentures, and requiring new conventional complete dentures regardless of the complexity of the case and the technique used. Participants were age 18 years or over, and able to give verbal and written consent, and be fluent in the English language to ensure adequate comprehension of the questionnaires to minimise data bias. Exclusion criteria included, patients who did not understand verbal and written English, and patients with Implant Supported Over dentures (ISODs), tooth supported over dentures or dentate patients. Patients with a history of Temporomandibular Disorders (TMDs) or jaw clenching were also excluded. These attributes were determined from the latest clinical examination noted in the patient's records. A positive ethical opinion from the National Research Ethics Services (NRES) committee, London-Westminster, approval number 15/LO/1299; August 2015, and a relevant R\&D (Research and Development) approval from Newcastle upon Tyne Hospitals (NHS Foundation Trust, number 7515, September 2015) were obtained.

\section{Study Participants}

Patients meeting the inclusion criteria, who were commencing treatment with conventional complete dentures, were approached at one of their treatment visits to the Prosthodontic clinic. Following a verbal explanation of the main aims of the study, potential participants were invited to take part in the study by giving them a participant information sheet, and were asked by the researcher to give verbal and written consent. Participants were asked to complete the ESIRE questionnaire before, and one month after treatment with conventional complete dentures. To avoid bias, administration of the questionnaires was undertaken by the researcher (HA), who was not involved with treatment. The sample size was determined using data on change in OHRQol one month following denture replacement (Ellis et al. 2007). Using paired $t$-test power calculation, it was estimated that 48 patients would provide $80 \%$ power to detect a mean pre-post treatment change of 5.5 ESIRE score points, at a 
significance level of $\alpha=0.05$. The aim was, therefore, to recruit approximately 80 patients with a target of 48 to complete the study based on refusal to participate and attrition.

\section{Data analysis}

For the quantitative data, an Excel database (Microsoft Office Professional Plus, 2013) was used to enter the raw data (scores) for the purpose of subsequent analysis using IBM SPSS Statistics 23.0 software (SPSS Inc., Chicago, IL, USA). For each participant the score for each domain was calculated by summing the scores of individual questions within that domain (answered on a VAS ranging from 0-100mm to give a score on 0-100) and dividing by the number of questions in that domain to give a domain score of 0-100. For each participant a total ESIRE score was obtained by summing the scores for each domain and dividing by the number of domains to give a final score of between 0-100. Descriptive analysis was performed using frequency measures for categorical variables (e.g., gender, age group, way of referral, regular dental visit, number of dentures used before and period of wearing dentures), and mean, median, standard deviation, standard error, percentiles, and confidence intervals for continuous variables (i.e., ESIRE score). Normality test (ShapiroWilk test), skewness, and kurtosis were used to test the normality of distribution of the data. In order to test the difference between the pre and post-treatment ESIRE scores, the assumption of normality was tested using the difference between paired scores, rather than the raw scores themselves based on the 'central limit theorem (CLT)' (Field 2013), which argues that this assumption is often satisfied for large data sets $(n>30)$. Paired t-test was used to determine the mean difference of total ESIRE score, before and after provision of new conventional complete dentures. The paired t-test was used to assess the mean differences of the scores for each domain of the ESIRE questionnaire. The independent sample t-test was used to determine gender differences in the change in ESIRE score after treatment. Sequential Bonferroni correction procedure (Holm 1979) was used to minimize the probability of 'Type 1' error (rejecting the null hypothesis when it is true). Cohen's standardized effect size (Cohen's $d$ ) was used to measure the effect size (ES) (the difference between two means expressed in standard deviation units). This was calculated by measuring the difference between the means of the two groups (pre-and post-treatment) divided by a standard deviation of group one (using pre-treatment ESIRE variance). Cohen's benchmarks (small: $\mathrm{ES}=0.2$, moderate: $\mathrm{ES}=0.5$, and large: $\mathrm{ES} \geq 0.8$ ) for interpreting the effect size were used to indicate the magnitude of change observed (Cohen 1988). Responsiveness to change (Deyo et al. 1991) was measured by comparing the pre and post-treatment ESIRE scores. A 
decrease in the effect of social and emotional issues around eating with dentures should generate an increase in the ESIRE scores, reflecting an improvement in ERQoL.

Responsiveness was initially investigated using the paired t-test to determine any difference in the mean of total scores, before and after provision of new conventional complete dentures (Deyo et al. 1991). Effect size was also used to evaluate the responsiveness of the ESIRE questionnaire (Cohen 1988). Responsiveness of the ESIRE questionnaire to changes in ERQoL was also quantified using a Standardised Response Mean (SRM) (Husted et al. 2000). Significance level was set at $p<0.05$.

Qualitative data from pre- and post-treatment ESIRE questionnaires were categorised into six main themes based on the six domains of the questionnaire. Indicative quotations from interviewees were coded according to study number; for example, (P01, P02...), gender; Male/Female (M/F) and age of participants during their recruitment to the study. 


\section{Results}

\section{Quantitative data (VAS scores)}

Table 1 illustrates characteristic of participants. Seventy-seven participants were recruited. Fifty participants aged 52-85 years (mean age, 72 years, with $46 \%>75$ years), 21 males (42\%), and 29 females (58\%) completed both questionnaires. Participants were either referred by their General Dental Practitioners (GDPs) from primary health care $(n=38(76 \%))$ or were self-referred $(\mathrm{n}=12(24 \%))$. At the time of recruitment, a large percentage of participants (84\%), had worn two or more set of dentures for three years or more.

Figure 1 shows descriptive statistics and distribution of the pre- and post-treatment ESIRE scores. Visual inspections of these box plots showed that the pre-treatment and post-treatment scores were approximately symmetrical with no obvious outliers. Overall, the post-treatment ESIRE scores were higher than the pre-treatment ESIRE scores. The mean scores were 39.91 for the pre-treatment questionnaire and 60.24 for the post-treatment.

Descriptive statistics of the difference between paired scores showed that the data were approximately normally distributed (Shapiro-Wilk test statistic $0.959, p>0.05$, and not skewed, z-value 1.83). In addition, the sampling distribution was approximately normally distributed for all domains. Total ESIRE score, and the scores for all domains, significantly increased following treatment (Table 2). The mean increase in score was $+20.33(p<0.001)$. Using a sequential Bonferroni correction test, all $P$-values were significant at a table wide 0.05 level.

Effect size of change in total ESIRE scores, and the score all domains is presented in Table 2. The effect size, as calculated by measuring Cohen's $d$, large (0.95) for the total score and ranged from medium $(0.59)$ for the domain relating to 'time for eating or preparation of meals' to large (1.30) for the domain relating to 'enjoyment of food/ eating' with the exception of the domain relating to 'interruption to meals', which was small (0.37). Values of SRM were also large (0.87) for the total score and ranged from medium (0.54) for the domain relating to 'time for eating or preparation of meals' to large (1.1) for the domain relating to 'enjoyment of food/ eating' with the exception of the domain relating to 'interruption to meals', which was small (0.36).

There was a non-significant trend $(\mathrm{P}=0.07)$ towards a greater increase in score in males compared with females (Table 3). No statistically, significant gender difference was observed 
for change in the following domains: enjoyment of food/eating, interruption to meals, confidence when eating and time for eating or preparation of meals. However, men showed a statistically significant greater increase for the domains of self-consciousness/embarrassment and functional ability to eat.

Figure 2 shows the percentage of pre-and post-treatment ESIRE scores classified as low, medium, or high. Following treatment, a lower proportion of scores were classified as low.

\section{Qualitative findings}

\section{Enjoyment of food/ eating}

Before treatment, the majority of participants commented that existing dentures negatively influenced enjoyment of eating and to a lesser extent drinking, particularly outside the home in public places or with relatives or friends. As one participant reported:

“Really badly, don't go for meals with my partner anymore. On holiday cannot eat what I want. Makes me depressed as I eat junk food and put weight on. I am constantly being told to lose weight by my consultant as it affects my condition.'”(P42, M63)

Other participants were embarrassed, felt a discomfort, and attributed this to poorly fitting dentures and chewing difficulty:

"I find eating in public embarrassing as the dentures tend to move about and I find biting into food impossible. Sometime food gets under the bottom dentures.' (P11, F69)

These sentiments are reflected by the low pre-treatment ESIRE score (Table 3).

When the patients were asked about their satisfaction with their ability to chew foods, some were dissatisfied mainly due to poor fit and instability of the dentures, particularly lower dentures. This affected their ability to chew hard or tough foods:

"Unable to chew an apple unless it is sliced or stewed. Top set is fine but bottom set keep rising when I chew and the food gets under the palate, so I have to remove the bottom set to enable me to eat my meal.", (P35, F74) 
However, chewing difficulty was less of an issue for other participants; one participant mentioned:

"I manage to chew pretty well with most foods." (P11, F69)

The ability to taste foods seemed to be not affected by wearing dentures for most participants, who were satisfied with their taste ability, as one participant reported:

"I am quite pleased with the taste of food, I am not sure whether false teeth make any difference with the taste of food." (P44, M69)

However, for some, taste was affected by inability to chew foods:

"Because I am not chewing my food properly, I swallow bigger amounts, so therefore I can't really taste my food the way I should."' (P23, F75)

After treatment, the most common response of most participants was achieving a degree of enjoyment of food or eating with their new dentures and this was reflected by the higher scores for this ESIRE domain that were achieved post rehabilitation (Table 2).

"Very good fit and comfortable. Able to taste food properly. Dentures fit well and don't move about when eating. Make it enjoyable when go out with friends and family.'”(P04, M65)

Despite the improvement of scores following treatment, and the positive comments of many patients regarding enjoyment of eating and drinking, some participants still had eating problems with their new dentures. For example, one participant mentioned a serious impact of new dentures on eating:

'Can only 'suck' my food then swallow if soft/ small enough. New dentures are only ok for cosmetic use. No eating, drinking sometimes ok. No real food eaten for 20 months. Very boring [for eating], some foods all the time dentures [are] out in a napkin. Some food gets stuck in my throat. So avoid lots of food on menu. Meat, vegetables, apples etc. any hard foods that you have to bite into. Unwell, not a balanced diet for ages. No pleasure to eat out or have a wide range of food." (P45, F67). 
It seems that despite improvement in the overall score of this domain, there is still a loss of enjoyment of foods during eating, possibly due to chewing difficulties.

\section{Self-consciousness/ embarrassment}

Before denture replacement, there were several examples where participants were extremely self-conscious or embarrassed because of their existing dentures during eating, and to a lesser extent, during drinking. One participant reported a typical story of the impact of complete dentures on social and emotional issues around eating with dentures:

“Because teeth drop and I start whistling, generally feel uncomfortable and embarrassed. Don't go out and eat in public at all. On holiday, take teeth out before eating carry them around, and moan to partner, everyone knows I have problems with teeth and people give advice, try to make jokes and live with it but I get depressed and frustrated.' (P42, M63)

After denture replacement, many participants described feelings of not being as selfconscious or as embarrassed as they had been before. A number of statements from the patients supported the improvement in the mean score of this domain (Table 2), for example:

“Not a lot with my new dentures.” (P32, M70)

“.... I have had no embarrassing moments really. ' (P12, F79)

Nonetheless, for some participants the experience of self-consciousness or embarrassment continued after treatment. One participant, who mentioned a similar story before and after denture replacement, pointed out a typical example of this:

"I look very old, ugly without the dentures in my mouth, but have to remove them if I want to eat in a cafe. Make me depressed, not wanting to go out for social events etc. Feel sick, older due to types of foods I eat/ swallow. Dentures only stay in if I do not move mouth muscles in any way, so only talk when denture in mouth not eat. Cannot eat anything, while denture in mouth.... Hide behind a napkin, as mouth is horrible, when trying to keep food inside, before able to swallow food can drop back into palate. Make me withdraw from many events in normal life, don't enjoy any food now, as 
bored having same food week after week for 20 months. Don't eat with dentures, I can't.' (P45, F67)

It appears that denture replacement can decrease the feelings of self-consciousness or embarrassment among some denture wearers. Nonetheless, for some, such feelings continue after denture replacement despite the significant overall improvement of the ESIRE score of this domain among the study population. Movement and falling out of the dentures during eating, and associated interruptions to meals could be reasons for this embarrassment.

\section{Interruption to meals}

Prior to the treatment, many respondents interrupted their meals to clean foods from their existing dentures. Several participants considered doing such process as annoying and embarrassing, particularly outside the home:

"The embarrassment of having to be excused when I leave the table to go to bathroom to clean the dentures."' (P35, F74)

"It is very annoying as you would have to make an excuse to leave the table way through a meal. If I try to manage until the meal is finished, I would often end up with an ulcer where food had irritated."' (P24, F65)

"It's fine when I am at home, but very awkward if I am out." (P29, F62)

After treatment with new dentures, there was indication that for some, interruption to meals was less of an issue that before, for example:

“This has not occurred with my new dentures.'”(P09, F75)

“This rarely happens and has really no effect on me. It just needs to be done." (P18, M82)

However, the smaller improvement in the score for this domain (Table 2) is reflected in the finding that some participants continued to experience interruption to meals to clean their new dentures from some foods (e.g., seeds and nuts). They described the process as annoying or upsetting especially if dentures have been fixed with fixative and food got underneath them: 
"It is only rarely when I eat tomatoes nuts and seeded bread. But I continue to try stick to my normal diet."' (P12, F79)

"It is very upsetting as you need to excuse yourself and it is obvious something is wrong. "’ (P24, F65)

"This is very uncomfortable and inconvenient especially with using a fixative." (P19, F77)

Although, for some patients, interruption to meals continued, adversely affecting confidence to eat in public places and in front friends and relatives, denture replacement reduced the interruption to meals for some, and overall, the score for this domain increased.

\section{Confidence when eating}

Several participants had experienced a lack of confidence during eating and drinking with their existing dentures before treatment because they had concerns that their dentures may fall down during eating or drinking. When participants were asked about their level of confidence when eating and drinking and what affected this, one participant replied:

"The fact that the denture will move and I will be unable to finish a meal. I do feel less confident when eating out and am often relieved when the meal is finished and I have manged.'”(P24, F65)

A lack of confidence during eating and drinking adversely affected food choice and social interaction with others:

"It limits my choice of foods, apples for example can only be eaten by cutting into small pieces with a knife."' (P46, M75)

"It makes me not want to go out for meals as I am always uneasy. In case anything gets stick or the denture drops. ', (P11, F69) 
Despite the overall improvement of the score of this domain (Table 2), many participants did not provide free text answers to explain their VAS scores. The level of confidence increased after dentures replacement for some participants, who became more confident with their new dentures; for example, one participant explained:

\section{“Lot more confident, now I have new dentures. ' (P32, M70)}

Other participants did not lack confidence, particularly at home, but many had concerns when eating outside their homes, suggesting that eating out remains a problem for them:

'Doesn't affect as much now [compared with when participant was 16 years of age].', (P15, M52)

“Doesn't affect me at home. Only when eating out as there might be a food on my teeth." (P50, F68)

The loss of confidence during eating with dentures can in part be attributed to movement and falling down of the dentures, which was regarded as an embarrassing issues. Likewise, the increase of confidence during eating may be attributed to the increase of fit and stability of the new dentures.

\section{Time for eating or preparation of meals}

Before treatment, participants described taking a long time to finish meals, meaning food became cold, and that they were the last person to finish a meal, which caused them embarrassment. These issues were regarded as a major concern by denture wearers. Many patients used denture fixatives or pastes to fix their dentures during eating to alleviate this. One participant reported:

'If I am still eating when others have finished they tend to watch me eating. I don't like to be the only one eating when others have finished I may be holding them back from the next course. I always ensure that my dentures are held in place as good as possible by applying a fixative,. ", (P46, M75)

After denture replacement, there was an improvement in the overall score of this domain (Table 2), and many patients seemed unconcerned about the time it took them to eat a meal: 
"I am not concerned at all. Sometimes with my old set, I might put some [fixative] on them. If it was an important do [special occasion]. ' (P44, M69)

However, some patients still had concerns about the time it took to eat a meal, and used fixatives or pastes to fix their new dentures during eating, particularly when eating out with company:

"In company, I sometimes leave my meal unfinished if I am slow. Sometimes it's cold before I am finished not a happy chappy. I always use a fixative when I eat in company, I couldn't eat without it.' '(P19, F77)

Using denture fixatives to fix new dentures during eating may be a means to increase confidence when eating but could also be an indicator of poor retention and fit of the dentures.

\section{Functional ability to eat}

Before treatment, there were many examples of patients, who were struggling to eat hard or tough foods (e.g., apples, steaks, seeds and nuts, sticky or chewy foods, and lettuce). One participant described almost all functional difficulties associated complete dentures:

"I could never bite into an apple, my teeth do not meet so I could not bite into anything. I would be scared I would snap a tooth this happened a couple of years ago. Pips and seeds get trapped under my denture and if I do not get them out immediately, I get ulcer. I can manage steak if it is extremely tender. I love nuts but like seeds they get caught under denture and ulcer are the result. Sticky or chewy foods would stick to denture and pull off fixative, so very uncomfortable. If I try to eat lettuce on its own, the teeth obviously do not meet properly at the back so it would be too thin to be eat." $(\mathrm{P} 24, \mathrm{~F} 65)$

Despite the significant improvement in overall score with regard to functional ability to eat (Table 2), several participants reported the same difficulties in eating hard foods after denture replacement. For example, one participant was experiencing continued eating difficulties with 
her new dentures, and mentioned a similar story to his experience with old dentures concerning the ability to eat hard or tough foods:

"I would never try to bite an apple as I know I would not be able to. Seeds get caught under denture. Steak would have to be very tender. Nuts gets caught and this hurts often causing ulcer. Sticky or chewy foods would stick to dentures and pull them apart. Lettuce is so thin and very hard to eat. I know that my new dentures is better than my old one. I still need paste and they are nothing like having your own teeth but they are better and once they have been altered they are catching I am sure I will be quite satisfied."' (P24, F65)

However, another participant commented that she could manage to eat some hard foods with their new dentures better than the old ones:

"I can chew steak better on one side. It does not take me as long as it used to. Find it easier to eat lettuce than before."' (P29, F62)

Despite the significant improvement in score for this domain, the functional ability to eat hard or tough foods is one of the main eating-related problems that was described by some denture wearers after provision of the new dentures.

Overall the qualitative findings revealed that denture replacement can positively improve the enjoyment of food/eating, socialising, self-consciousness, interruption to meals, comfort and function. However, for some denture wearers, wearing complete dentures still has a negative effect on social and emotional issues around eating even after denture replacement. 


\section{Discussion}

To the authors' knowledge, this is the first study that has longitudinally followed the effect of denture replacement on ERQoL. In general, findings showed an increase in ESIRE score following treatment indicating more positive ERQoL. Improvement in ERQoL related to all ESIRE domains. Based on the assumption that using multiple t-tests on small sample could lead to loss of a statistical power each time the test repeated (Holm 1979), the sequential Bonferroni correction procedure was applied to minimize the probably of getting a significant result purely by chance. Using this formula, all p-value were significant at a table wide $\mathrm{p}<0.05$. The findings reject the null hypothesis $(\mathrm{Ho})$ (there would be no change in ERQoL, from before to after denture replacement), and support the alternative hypothesis $\left(\mathrm{H}_{1}\right)$ (there would be a change in ERQoL, after treatment with new conventional complete dentures).

The significant improvement in post-treatment ESIRE scores could be attributed to several factors: first, the presumption that denture replacement can enhance retention, stability, and occlusion; hence, improve chewing, and subsequently patient's satisfaction. Newly fitted dentures could have optimal retention and stability (Bartlett et al. 2013), which could have a positive influence on using dentures for eating (Fenlon and Sherriff 2008). In addition, social and emotional instability of patients were probably resolved by replacement dentures with good fit and retention, consequently increased satisfaction might be responsible for improvement in scores (Demers et al. 1986; Sheiham et al. 2001). Second, most participants were referred to the dental hospital by their GDPs from dental practices and were therefore more likely to be experiencing particular difficulty with their dentures; hence, such patients could feel a more positive impact after denture replacement due to significant improvements from enhanced retention, stability, and carefully prescribed occlusion schemes. Research shows that patients' satisfaction after a dental treatment is likely depend on their expectations before the treatment (Smith and McCord 2004); hence, it could be argued that participants in the present study had low expectations because most of them had been referred by their GDPs due to previous denture-related problems. This could influence their responses to the follow up ESIRE questionnaire. However, it is not uncommon to have a response bias in studies involving completion of self-administration questionnaires. Response bias is a generic term used when participants do not give an accurate or honest response during completion of self-administrated questionnaire (Furnham 1986). Respondents in studies, which involve selfadministration questionnaires, may only select the most extreme choices or answers offered (Meisenberg and Williams 2008) or they could think that they should answer the questions 
according the researcher's inclination (Davis et al. 2000). There is always a risk that people, who come to a clinic and get their treatment (e.g., complete dentures) free of charge (which is the case in the UK) will, feel grateful and then want to give a positive scenario or feedback about their treatment experience. To minimise the effect of response bias, the research student asked and encouraged all participants to answer the questions honestly, in their home, in their own time, to maintain independency in expressing their own opinions. Moreover, sealable envelopes were provided to all participants for completed questionnaires to ensure that the dental team, who were responsible for the treatment had no access to the participant' responses. Denture satisfaction is likely to be influenced by the prosthodontic experience of clinicians (Kimoto et al. 2013). Such influences cannot be ruled out as a factor contributing towards increased patient satisfaction with the treatment. Research has shown new complete dentures finished by undergraduate dental students are technically satisfactory, in terms of fit and occlusion (Davis et al. 1986). These previous findings could be reflected in the present study, in which undergraduate students, who were supervised by clinicians with good clinical experience, did all treatments.

Measuring the size of change in ERQoL is essential in order to get some practical idea about the clinical significance of the results. Cohen's standardized effect size (Cohen's $d$ ) is widely used as a mean to detect 'the clinical meaningfulness' (Allen and McMillan 2003; Kuo et al. 2013), and was used in the current study. The effect size was either medium or large for all domains of the ESIRE questionnaire; thus, the improvements were clinically significant. Due to the difference in study design, aims, objectives, age and sample size, it was difficult to compare the effect size of the present study with other studies. However, the effect size was similar to the findings of Allen and McMillan (Allen and McMillan 2003), who demonstrated a moderate to large effect size for change in OHIP (Oral Health Impact Profile) score for patients treated with conventional complete dentures in an hospital environment. In the current study, the effect size was greater than previous studies that used OHIP-EDENT (Heydecke et al. 2003; Kuo et al. 2013).

For the majority of ESIRE domains, there were no between gender differences in change in ESIRE scores, suggesting that prosthetic rehabilitation improves ERQoL to a similar extent regardless of gender. However, the statistically significant between gender difference in the improvement of scores for self-consciousness/ embarrassment and functional ability to eat shows that improvements were less for women. These differences resulted in a nonsignificant trend towards a greater increase in total ESIRE score for men overall. These 
findings differ from previous research concluding that gender difference has no impact on patient satisfaction and quality of life of complete denture wearers (Geckili et al. 2012). The current findings suggest that prosthetic rehabilitation is less able to reduce self-conscious or embarrassment and to overcome functional difficulties for women compared with men.

Women being more self-conscious when eating with dentures resulting in greater embarrassment in social life than male patients, may explain this (Hurd 2000; Trulsson et al. 2002). Moreover, women may wish to eat foods that are more functionally difficult to eat compared with the foods that men wish to eat, although this theory warrants further investigation. The findings of the gender differences are in agreement with results of Pan et al. (2008), who reported that older men were more satisfied with conventional dentures than older women in terms of aesthetics and ability to chew. Although it has been reported that women show more improvement in overall OHRQoL after complete denture therapy (Sivakumar et al. 2015), the current findings suggest that might be due to impacts on quality of life factors other than functional ability to eat foods and self-consciousness on eating.

A secondary aim of this research was to measure the responsiveness of the ESIRE questionnaire. Responsiveness to change of oral health instruments is often assessed by using different measures such as effect sizes for the change in score, the minimal importance difference, the standardised response mean and Guyatt's responsiveness index (Locker et al. 2004). In this study, effect size and SRM were used to provide a first data on the responsiveness of the ESIRE questionnaire to change in ERQoL. If the ESIRE questionnaire is to function as an outcome measure for use in evaluating changes in ERQoL and monitoring patients it should also be responsive to changes in health status over time. Results of this study showed that the ESIRE questionnaire was responsive to change in ERQoL among the sample of denture wearers.

No previous studies have prospectively investigated the impact of denture replacement on ERQoL. However, the quantitative data from the present study concur with data from other studies, which used OHIP-EDENT to measure changes in OHRQoL, including issues around eating after denture replacement. For example Viola et al. (2013) and Sivakumar et al. (2015) reported a significant improvement in patient's satisfaction and OHRQoL among patients treated with new conventional complete dentures. However, the findings are in contrast to other studies (AlBaker 2013; Allen 2005) reporting no improvement in patient's satisfaction and OHRQoL after denture replacement. 


\section{Strengths and limitations}

The study had some methodological limitations to acknowledge. First, it must be acknowledged that dental hospital patients may not be typically of the edentulous population receiving replacement dentures. Moreover, all patients were from the Northeast of England, which could mean that the data are not widely generalizable. Nonetheless, it could also be argued that the results are applicable globally, to groups of people, who have comparable sociodemographic features and clinical profiles.

In the present study, participants completed the follow up ESIRE questionnaire approximately one month after provision of new dentures. One month after treatment with new dentures has been used as a follow up period in previous studies evaluating elements of OHRQoL, and patient's satisfaction (Ellis et al. 2007; Kimoto et al. 2013; Sivakumar et al. 2015). However, the effect of denture replacement on the quality of life and patient's satisfaction could change over a longer period of time (Reissmann et al. 2016). A larger improvement in ERQoL may have been observed after all the adjustments are completed and the patient has further adjusted to their dentures. Thus, it might have been desirable to follow up the same participants for longer (i.e., three months, six months, and after one year); nevertheless, limited resources and time scale of this study did not enable this.

\section{Conclusion}

The study data provide the first evidence of the responsiveness of the ESIRE questionnaire to changes in ERQoL among a population of conventional denture wearers. The quantitative and qualitative findings show denture replacement can directly improve ERQoL highlighting a benefit of using conventional compete dentures as a treatment option for edentulous patients. The highly significant improvement in the ESIRE scores indicated 'clinical meaningfulness' of the effect of denture replacement on ERQoL. However, the qualitative findings showed that for some, negative impacts on eating persisted after denture replacement indicating that providing eating advice for such patents is warranted.

\section{Author Contributions}

H,F. Al-Sultani contributed to the conception, design, data acquisition, data analysis, and interpretation, drafted and critically revised the manuscript; J.C. Field, contributed to the conception, design, data analysis, and interpretation and critically revised the manuscript; J.M. Thomason, contributed to conception, design and critically revised the manuscript; P.J. 
Moynihan contributed to conception, design, data analysis and critically revised the manuscript. All authors gave final approval and agree to be accountable for all aspects of the work.

\section{Acknowledgements}

This work was funded by Iraqi Ministry of Higher Education and Scientific Research (MoHESR). The authors would like to thank all the patients, who participated in this study, all dental students and dental staff, who provided treatment to the patients at Newcastle Dental hospital, UK for their help and support. The authors would like to recognize Professor Janice Ellis, Professor Justin Durham, Dr Ian Ellis and Dr Kim Pearce (Statistician) for their continuous and excellent support. The authors declare no potential conflicts of interest with respect to the authorship and/or publication of this article. 


\section{References:}

AlBaker AM. 2013. The oral health-related quality of life in edentulous patients treated with conventional complete dentures. Gerodontology. 30(1):61-66.

Allen PF. 2005. Association between diet, social resources and oral health related quality of life in edentulous patients. Journal of oral rehabilitation. 32(9):623-628.

Allen PF, McMillan AS. 2003. A longitudinal study of quality of life outcomes in older adults requesting implant prostheses and complete removable dentures. Clin Oral Implants Res. 14(2):173-179.

Bartlett DW, Maggio B, Targett D, Fenlon MR, Thomas J. 2013. A preliminary investigation into the use of denture adhesives combined with dietary advice to improve diets in complete denture wearers. Journal of dentistry. 41(2):143-147.

Basker RM, Davenport JC, Thomason JM. 2011. Prosthetic treatment of the edentulous patient. John Wiley \& Sons.

Carlsson GE, Omar R. 2010. The future of complete dentures in oral rehabilitation. A critical review. Journal of oral rehabilitation. 37(2):143-156.

Cohen J. 1988. Statistical power analysis for the behavior science. Lawrance Eribaum Association.

Davis DM, Fiske J, Scott B, Radford DR. 2000. Prosthetics: The emotional effects of tooth loss: A preliminary quantitative study. British Dental Journal. 188(9):503-506.

Davis EL, Albino JE, Tedesco LA, Portenoy BS, Ortman LF. 1986. Expectations and satisfaction of denture patients in a university clinic. The Journal of Prosthetic Dentistry. 55(1):59-63.

Demers M, Brodeur JM, Simard P, Vallee R. 1986. Problems associated with edentulism among the elderly. Journal (Canadian Dental Association). 52(12):1019.

Deyo RA, Diehr P, Patrick DL. 1991. Reproducibility and responsiveness of health status measures statistics and strategies for evaluation. Controlled clinical trials. 12(4):S142-S158.

Ellis JS, Elfeky AF, Moynihan PJ, Seal C, Hyland RM, Thomason M. 2010. The impact of dietary advice on edentulous adults' denture satisfaction and oral health-related quality of life 6 months after intervention. Clin Oral Implants Res. 21(4):386-391.

Ellis JS, Pelekis ND, Thomason JM. 2007. Conventional rehabilitation of edentulous patients: The impact on oral health-related quality of life and patient satisfaction. Journal of prosthodontics : official journal of the American College of Prosthodontists. 16(1):37-42.

Emami E, de Souza RF, Kabawat M, Feine JS. 2013. The impact of edentulism on oral and general health. International journal of dentistry. 2013.

Fenlon MR, Sherriff M. 2008. An investigation of factors influencing patients' satisfaction with new complete dentures using structural equation modelling. Journal of dentistry. 36(6):427-434.

Field A. 2013. Discovering statistics using ibm spss statistics. Sage.

Forgie AH, Scott BJJ, Davis DM. 2005. A study to compare the oral health impact profile and satisfaction before and after having replacement complete dentures in england and scotland. Gerodontology. 22(3):137-142.

Furnham A. 1986. Response bias, social desirability and dissimulation. Personality and Individual Differences. 7(3):385-400.

Geckili O, Bilhan H, Mumcu E, Dayan C, Yabul A, Tuncer N. 2012. Comparison of patient satisfaction, quality of life, and bite force between elderly edentulous patients wearing mandibular two implant-supported overdentures and conventional complete dentures after 4 years. Special Care in Dentistry. 32(4):136-141.

Guyatt G, Walter S, Norman G. 1987. Measuring change over time: Assessing the usefulness of evaluative instruments. Journal of Chronic Diseases. 40(2):171-178. 
Heydecke G, Locker D, Awad MA, Lund JP, Feine JS. 2003. Oral and general health-related quality of life with conventional and implant dentures. Community dentistry and oral epidemiology. 31(3):161-168.

Holm S. 1979. A simple sequentially rejective multiple test procedure. Scandinavian journal of statistics.65-70.

Hurd LC. 2000. Older women's body image and embodied experience: An exploration. Journal of Women \& Aging. 12(3-4):77-97.

Husted JA, Cook RJ, Farewell VT, Gladman DD. 2000. Methods for assessing responsiveness: A critical review and recommendations. Journal of clinical epidemiology. 53(5):459-468.

Hyland R, Ellis J, Thomason M, El-Feky A, Moynihan P. 2009. A qualitative study on patient perspectives of how conventional and implant-supported dentures affect eating. Journal of dentistry. 37(9):718-723.

Kelly SAM, Hyland RM, Ellis JS, Thomason JM, Moynihan PJ. 2012. Development of a patient-based questionnaire about emotional and social issues related to eating with dentures. Journal of dentistry. 40(8):678-685.

Kimoto S, Kimoto K, Kitamura A, Saita M, lijima M, Kawai Y. 2013. Effect of dentist's clinical experience on treatment satisfaction of a complete denture. Journal of oral rehabilitation. 40(12):940947.

Krunić N, Kostić M, Petrović M, Igić M. 2015. Oral health-related quality of life of edentulous patients after complete dentures relining. Vojnosanitetski pregled. 72(4):307-311.

Kuo H-C, Kuo Y-S, Lee IC, Wang J-C, Yang Y-H. 2013. The association of responsiveness in oral and general health-related quality of life with patients' satisfaction of new complete dentures. Quality of Life Research. 22(7):1665-1674.

Locker D, Jokovic A, Clarke M. 2004. Assessing the responsiveness of measures of oral health-related quality of life. Community dentistry and oral epidemiology. 32(1):10-18.

Meisenberg G, Williams A. 2008. Are acquiescent and extreme response styles related to low intelligence and education? Personality and Individual Differences. 44(7):1539-1550.

Moynihan P, Thomason M, Walls A, Gray-Donald K, Morais JA, Ghanem H, Wollin S, Ellis J, Steele J, Lund J. 2009. Researching the impact of oral health on diet and nutritional status: Methodological issues. Journal of Dentistry. 37(4):237-249.

Müller F. 2014. Interventions for edentate elders-what is the evidence? Gerodontology. 31(s1):44-51.

Pan S, Awad M, Thomason JM, Dufresne E, Kobayashi T, Kimoto S, Wollin SD, Feine JS. 2008. Sex differences in denture satisfaction. journal of dentistry. 36(5):301-308.

Reissmann DR, John MT, Feuerstahler L, Baba K, Szabó G, Čelebić A, Waller N. 2016. Longitudinal measurement invariance in prospective oral health-related quality of life assessment. Health and Quality of Life Outcomes. 14(1):88.

Scott BJJ, Forgie AH, Davis DM. 2006. A study to compare the oral health impact profile and satisfaction before and after having replacement complete dentures constructed by either the copy or the conventional technique. Gerodontology. 23(2):79-86.

Sheiham A, Steele JG, Marcenes W, Tsakos G, Finch S, Walls AWG. 2001. Prevalence of impacts of dental and oral disorders and their effects on eating among older people; a national survey in great britain. Community Dentistry and Oral Epidemiology. 29(3):195-203.

Sivakumar I, Sajjan S, Ramaraju AV, Rao B. 2015. Changes in oral health-related quality of life in elderly edentulous patients after complete denture therapy and possible role of their initial expectation: A follow-up study. Journal of Prosthodontics. 24(6):452-456.

Smith PW, McCord JF. 2004. What do patients expect from complete dentures? J Dent. 32(1):3-7.

Trulsson U, Engstrand P, Berggren U, Nannmark U, Brånemark PI. 2002. Edentulousness and oral rehabilitation: Experiences from the patients' perspective. European journal of oral sciences. 110(6):417-424. 
Viola AP, Takamiya AS, Monteiro DR, Barbosa DB. 2013. Oral health-related quality of life and satisfaction before and after treatment with complete dentures in a dental school in brazil. Journal of prosthodontic research. 57(1):36-41.

Tables and figures

Table 1: Participant characteristics: distribution of participants by age, gender, way of referral, regular dental visit, number of dentures used before, and period of wearing dentures.

\begin{tabular}{lcc}
\hline Variables & Number & Frequency (\%) \\
\hline Gender & 21 & \\
Male & 29 & $42 \%$ \\
Female & & $58 \%$ \\
\hline Age group (Years) & 8 & $16 \%$ \\
$<65$ & 19 & $38 \%$ \\
$\geq 65-74$ & 23 & $46 \%$ \\
$\geq 75$ & & \\
\hline Way of referral & 38 & $76 \%$ \\
Referred by GDPs & 12 & $24 \%$ \\
Self-referral & & \\
Regular dental visit & 37 & $74 \%$ \\
Yes & 13 & $26 \%$ \\
No & & \\
\hline Number of dentures used before & 8 & $16 \%$ \\
$<2$ dentures & 42 & $84 \%$ \\
$\geq 2$ dentures & & $16 \%$ \\
\hline Period of wearing dentures & 8 & $84 \%$ \\
$<3$ years & 42 & \\
$\geq 3$ years & & \\
\hline
\end{tabular}


Table 2: ESIRE scores before and following complete replacement dentures. Descriptive statistics for total score and scores for each domain.

\begin{tabular}{|c|c|c|c|c|c|c|c|}
\hline \multirow[t]{2}{*}{ Domain } & \multicolumn{2}{|c|}{$\begin{array}{c}\text { ESIRE scores-Mean } \\
\text { (SD) }\end{array}$} & \multirow{2}{*}{$\begin{array}{c}\text { Mean } \\
\text { difference } \\
\text { (SE) }\end{array}$} & \multicolumn{2}{|c|}{$95 \% \mathrm{CI}^{+}$} & \multirow[t]{2}{*}{$\begin{array}{l}\text { Paired-t } \\
\text { test }\end{array}$} & \multirow[t]{2}{*}{$\begin{array}{c}\text { Effect } \\
\text { size }\end{array}$} \\
\hline & $\begin{array}{c}\text { Pre- } \\
\text { treatment }\end{array}$ & $\begin{array}{c}\text { Post- } \\
\text { treatment }\end{array}$ & & Lower & Upper & & \\
\hline $\begin{array}{l}\text { Enjoyment of } \\
\text { food/ eating }\end{array}$ & $\begin{array}{l}33.87 \\
(21.02)\end{array}$ & $\begin{array}{l}61.15 \\
(24.89)\end{array}$ & $\begin{array}{l}27.28 \\
(3.63)\end{array}$ & 19.99 & 34.56 & $P<0.001 *$ & $\begin{array}{l}1.30^{\mathrm{a}} \\
1.1^{\mathrm{b}}\end{array}$ \\
\hline $\begin{array}{l}\text { Self- } \\
\text { consciousness/ } \\
\text { embarrassment }\end{array}$ & $\begin{array}{l}45.04 \\
(24.52)\end{array}$ & $\begin{array}{l}63.18 \\
(28.95)\end{array}$ & $\begin{array}{l}18.13 \\
(3.88)\end{array}$ & 10.35 & 25.92 & $P<0.001 *$ & $\begin{array}{l}0.74^{\mathrm{a}} \\
0.66^{\mathrm{b}}\end{array}$ \\
\hline $\begin{array}{l}\text { Interruption to } \\
\text { meals }\end{array}$ & $\begin{array}{c}45.80 \\
(33.69)\end{array}$ & $\begin{array}{l}59.08 \\
(34.29)\end{array}$ & $\begin{array}{l}13.29 \\
(5.27)\end{array}$ & 2.69 & 23.88 & $P<0.05^{*}$ & $\begin{array}{l}0.37^{\mathrm{a}} \\
0.36^{\mathrm{b}}\end{array}$ \\
\hline $\begin{array}{l}\text { Confidence when } \\
\text { eating }\end{array}$ & $\begin{array}{l}52.66 \\
(28.93)\end{array}$ & $\begin{array}{l}71.39 \\
(25.67)\end{array}$ & $\begin{array}{l}18.73 \\
(4.84)\end{array}$ & 9.01 & 28.45 & $P<0.001 *$ & $\begin{array}{l}0.65^{\mathrm{a}} \\
0.55^{\mathrm{b}}\end{array}$ \\
\hline $\begin{array}{l}\text { Time for eating } \\
\text { or preparation of } \\
\text { meals }\end{array}$ & $\begin{array}{c}46.47 \\
(31.18)\end{array}$ & $\begin{array}{c}64.94 \\
(30.08)\end{array}$ & $\begin{array}{l}18.47 \\
(4.85)\end{array}$ & 8.73 & 28.22 & $P<0.001 *$ & $\begin{array}{l}0.59^{\mathrm{a}} \\
0.54^{\mathrm{b}}\end{array}$ \\
\hline $\begin{array}{l}\text { Functional ability } \\
\text { to eat }\end{array}$ & $\begin{array}{l}25.71 \\
(20.42)\end{array}$ & $\begin{array}{c}43.87 \\
(28.59)\end{array}$ & $\begin{array}{l}18.16 \\
(3.67)\end{array}$ & 10.78 & 25.54 & $P<0.001 *$ & $\begin{array}{l}0.89^{\mathrm{a}} \\
0.70^{\mathrm{b}}\end{array}$ \\
\hline Total score & $\begin{array}{l}39.91 \\
(21.36)\end{array}$ & $\begin{array}{c}60.24 \\
(24.22)\end{array}$ & $\begin{array}{l}20.32 \\
(3.30)\end{array}$ & 13.69 & 26.95 & $P<0.001^{*}$ & $\begin{array}{l}0.95^{\mathrm{a}} \\
0.87^{\mathrm{b}}\end{array}$ \\
\hline
\end{tabular}

${ }^{+} 95 \%$ Confidence Interval of the Difference. $P<0.05$ denotes significance. $P<0.001$ denotes highly significance. $* P$ value is significant at table wide 0.05 level using a sequential Bonferroni correction procedure. Effect size (small $=0.2$ ), (moderate $=0.5$ ), (large $\geq 0.8$ ) measured using Cohen's $d$ using pre-treatment ESIRE variance ${ }^{\mathrm{a}}$, and $\mathrm{SRM}^{\mathrm{b}}$. 
Table 3: Gender comparison of change in the ESIRE score one month following treatment.

\begin{tabular}{|c|c|c|c|}
\hline \multirow[b]{2}{*}{ Domain } & \multicolumn{3}{|c|}{$\begin{array}{l}\text { Change in the ESIRE score between pre- and post- } \\
\text { treatment }\end{array}$} \\
\hline & $\begin{array}{c}\text { Male }(\mathrm{n}=21) \\
\operatorname{Mean}(\mathrm{SE})\end{array}$ & $\begin{array}{l}\text { Female }(\mathbf{n}=29) \\
\text { Mean (SE) }\end{array}$ & ${ }^{\text {a } P \text {-value }}$ \\
\hline Enjoyment of food/ eating & $32.76(6.00)$ & $23.31(4.43)$ & 0.20 \\
\hline $\begin{array}{l}\text { Self-consciousness/ } \\
\text { embarrassment }\end{array}$ & $27.71(6.13)$ & $11.28(4.67)$ & 0.04 \\
\hline Interruption to meals & $23.10(7.43)$ & $6.34(6.91)$ & 0.11 \\
\hline Confidence when eating & $23.67(8.48)$ & $15.31(5.70)$ & 0.40 \\
\hline $\begin{array}{l}\text { Time for eating or } \\
\text { preparation of meals }\end{array}$ & $19.95(8.16)$ & $17.48(6.05)$ & 0.81 \\
\hline Functional ability to eat & $27.38(6.39)$ & $11.45(3.97)$ & 0.03 \\
\hline Total score & $27.29(5.76)$ & $15.31(3.65)$ & 0.07 \\
\hline
\end{tabular}


Figure 1: Box plots illustrating descriptive statistics (median and IQR) and range of pre- and post-treatment total ESIRE score.

Figure 2: Percentage of pre- and post-treatment ESIRE scores classified as low, medium, and high, before and one month following complete denture replacement $(\mathbf{n}=\mathbf{5 0})$. 